\title{
Analysis of Active Flow Control Application in Subsonic Intake Integrated with Aircraft Glider
}

\author{
D.A. Luybimov ${ }^{1}$, A.A. Terehova ${ }^{1}$, M.E. Pikalov ${ }^{1}$, A.A. Postnikov ${ }^{1}$, \\ A.A. Frolov ${ }^{1}$, A.S. Skryabin ${ }^{2}$ \\ ${ }^{1}$ Central Institute of Aviation Motors, Moscow 111116, Russian Federation \\ ${ }^{2}$ Bauman Moscow State Technical University, Moscow 105005, Russian Federation \\ Postaa@rambler.ru
}

\begin{abstract}
This paper presents the study of subsonic intake, integrated with curvilinear fuselage fore body. Intake's complex shape leads to the formation of longitudinal vortices causing a significant uneven distribution of flow parameters at the output section of the intake. Numerical research, using ESI RANS and RANS / ILES methods, of air intake flow were obtain at different throttle modes. The flow fields were compare with the experimental results. Throttling modes with pronounced unevenness at the output section were determined. By means of RANS/ILES methods conducted study of a flow control with synthetic jets in the subsonic intake. The jet's parameters, which made it possible to reduce the circumferential unevenness by $5 \div 39 \%$ at the output section of the intake, were determined.
\end{abstract}

Keywords: Synthetic jets, aircraft glider, throttle performance.

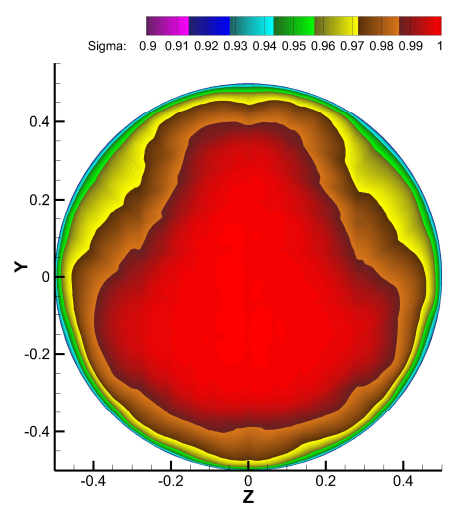

$a$

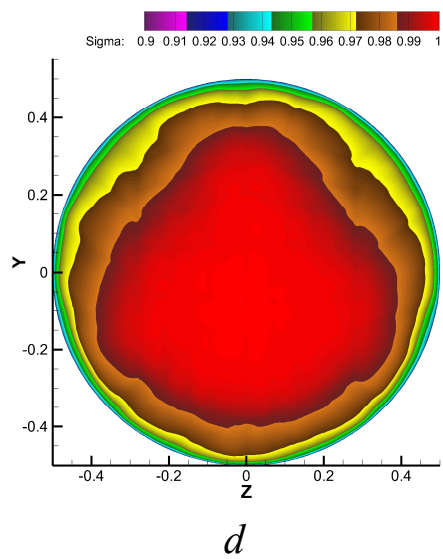

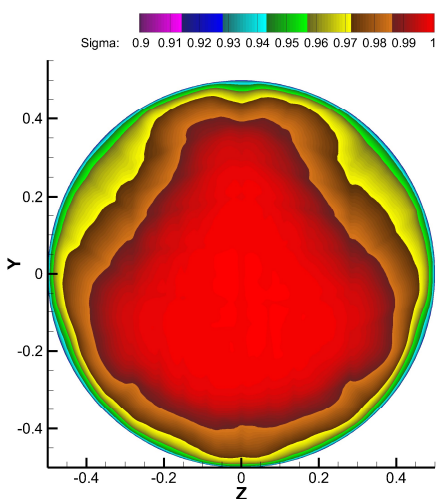

$b$

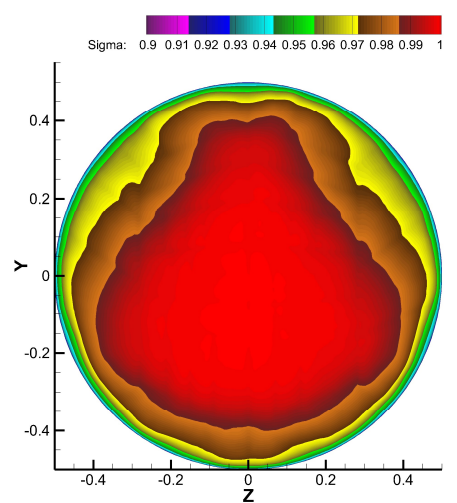

$c$

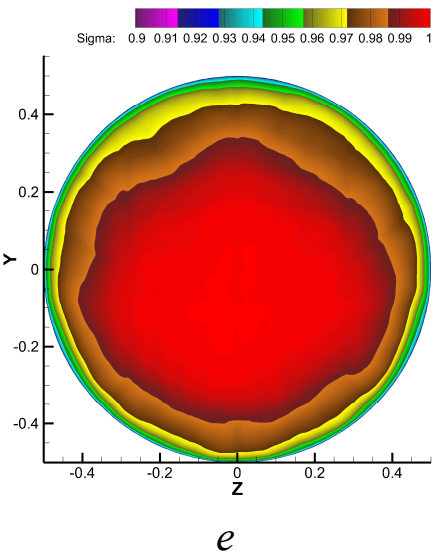

Synthetic jet's influence on output parameters of subsonic intake 


\title{
Анализ применения активного управления течением в дозвуковом пространственном воздухозаборнике в компоновке с планером летательного аппарата
}

\author{
Д.А. Любимов ${ }^{1}$, А.А. Терехова ${ }^{1}$, М.Е. Пикалов ${ }^{1}$, А.А. Постников ${ }^{1}$, \\ А.А. Фролов ${ }^{1}$, А.С. Скрябин ${ }^{2}$ \\ ${ }^{1}$ Центральный институт авиационного моторостроения, \\ Россия, г. Москва, 111116, Россия, Москва, ул. Авиамоторная, 2 \\ ${ }^{2}$ Московский государственный технический университет им. Н.Э. Баумана, \\ Россия, г. Москва, 2-я Бауманская ул., д. 5, стр. 1 \\ Postaa@rambler.ru
}

\begin{abstract}
Аннотация
Исследовано течение в воздухозаборнике (В3) силовой установки, интегрированной с фюзеляжем летательного аппарата (ЛА). Сложная форма B3, переход от D-образного поперечного сечения на входе к круглому на выходе, приводит к образованию продольных вихрей, вызывающих значительную неравномерность распределения параметров течения в выходном сечении В3. Проведены расчеты с помощью ESI RANS и RANS/ILES методов на различных режимах дросселирования. Выполнено сравнение полей течения с результатами эксперимента. На режиме дросселирования с выраженной неравномерностью полей полного давления на выходе из B3 RANS/ILES методом проведено исследование газодинамического управления течением с помощью синтетических струй на течение в В3. Определены режимные параметры струй, позволивший уменьшить окружную неравномерности на $5 \div 39 \%$ на выходе из канала.
\end{abstract}

Ключевые слова: Синтетические струи, планер ЛА, дроссельные характеристики.

\section{1. Введение}

При разработке новых летательных аппаратов (ЛА) перспективным считается интеграция силовой установки (СУ) с планером летательного аппарата. Среди прочих рассматриваются силовые установки, расположенные над планером. Причины такого расположения могут быть различны. Например, уменьшение шума силовой установки на взлете, особенности поверхности, с которой взлетает самолет, а также беспилотные ЛА, у которых силовая установка по компоновочным соображениям интегрирована с планером.

Примером может служить коммерческий гражданский самолет [1]. Компоновка этого летательного аппарат включает в себя интеграцию D-образного входного устройства, расположенного в хвостовой части на клине слива. Сложная геометрия такого В3 приводит к большой неравномерности, течения в самом канале и на выходе из него [2]. Для улучшения характеристик подобных диффузоров применяются различные способы - пассивные и активные методы управления течением. К пассивным методам относятся разнообразные завихрители и турбулизаторы, к активным - вдув и выдув микроструй, отсос воздуха перед зоной отрыва и т.д. Перспективным является управление течением с помощью так называемых синтетических струй, работа которых сводится к чередующемуся выдуву газа в поток из замкнутой полости 
за счет изменения ее объема, с последующим всасыванием в нее низкоэнергетического внешнего потока. Примеры их использования для управления течением в В3 приведены в $[3,4]$.

В настоящей работе рассмотрен вариант расположения СУ над фюзеляжем дозвукового ЛF в непосредственной близости к нему. Форма фюзеляжа ЛА такова, что В3 оказывается в «тени» его носовой части. Ранее были проведены экспериментальные и численные исследования этого В3 [5]. На основании полученных результатов был выбран интересный для практических приложений режим, на котором проводилось сравнение с экспериментом расчетов, полученных с помощью RANS методов в пакете ESI - Software [6], и расчетов, полученных с помощью RANS/ILES метода высокого разрешения [7]. Была исследована возможность применения синтетических струй для управления течением в В3 и улучшения его характеристик.

Целью настоящей работы является оценка точности расчетов течения в указанном В3 с помощью различных численных методов и анализ эффективности применения синтетических струй для улучшения его характеристик В3.

Задачами в рамках поставленной цели являются:

- валидация ESI RANS и RANS/ILES метода на выбранном режиме дросселирования;

- расчет и анализ характеристик течения в В3 на различных режимах работы синтетических струй.

\section{2. Геометрия B3, режимные параметры и граничные условия}

На рис. 1 представлена принципиальная схема расчетной области (2). Ее размеры с размещенным В3 диаметра $d y$ в компоновке с фюзеляжем ЛА (3), составляют от 5 до 10 калибров $d_{y}$ вдоль разных осей координат. Большие размеры расчетной области позволяют уменьшить влияние использованных упрощенных граничных условий. Для более точного описания течения строилась неравномерная сетка с увеличенным числом ячеек в пристеночной области потока около обтекаемой модели. Общее количество гексагональных ячеек в поле течения составляло $6 \times 10^{6}$.

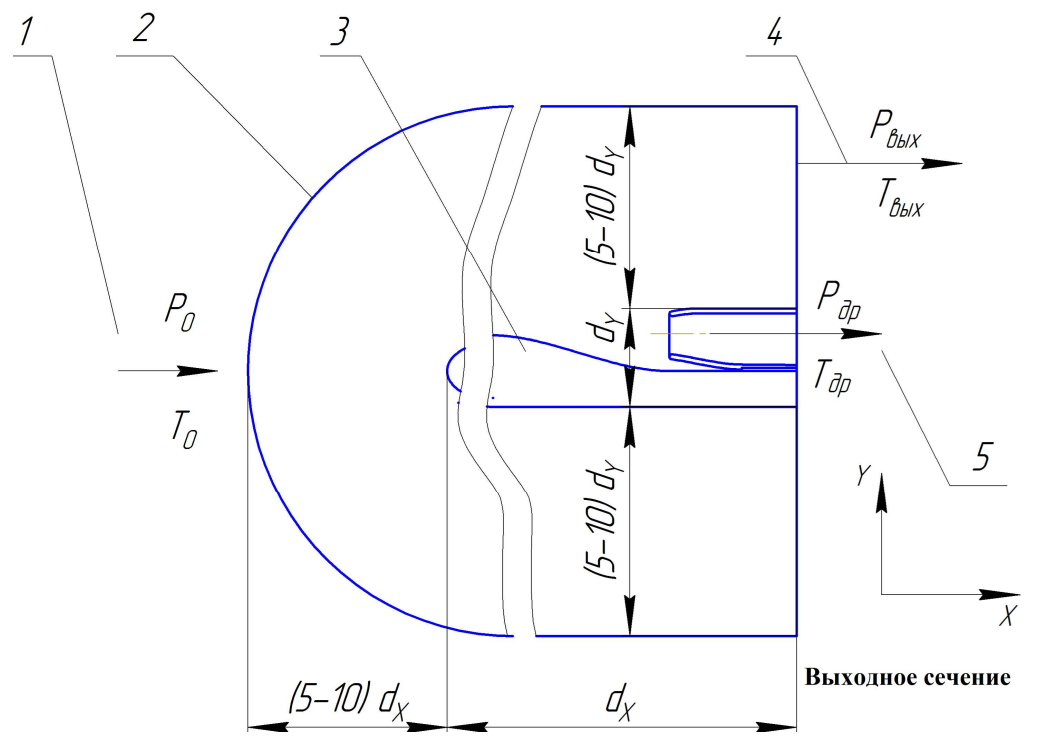

Рис. 1. Принципиальная схема расчетной области и задание граничных условий: 1 - входные параметры; 2 - границы расчетной области; 3 - исследуемая модель; 4 - выходные параметры в области внешнего обтекания; 5 - параметр на выходе из B3, задаваемые при изменении степени дросселирования

При расчете RANS/ILES методом в области грубой сетки и около стенок канала течение описывается с помощью нестационарного метода RANS - URANS с полуэмпирической моде- 
лью турбулентности Спаларта - Аллмараса, а в области отрывного течения, где была использована мелкая сетка, с помощью метода LES с неявной подсеточной моделью - ILES. Подобные комбинированные методы позволяют существенно повысить точность описания отрывных течений.

Расчеты RANS выполнялись с помощью программного комплекса ESI - Software [3], ocнованного на решении стационарных уравнений Навье - Стокса, осредненных по Рейнольдсу для трехмерных вязких течений.

Граничные условия задавались в соответствии с параметрами, которые обеспечивались в аэродинамической трубе. На входе в канал задавались полные параметры потока: давление $P_{0}=1 \times 10^{5}$ Па и температура $T_{0}=300 \mathrm{~K}$. Выходные параметры $P_{\text {вых }}$ и $T_{\text {вых }}$ соответствовали значениям во внешнем потоке на рассматриваемом режиме полета $\left(\mathrm{M}_{0}=0.4\right)$. Условия дросселирования В3 задавались величинами $P_{\text {др }}$ и $T_{\text {др }}$.

При проведении расчетов, для обеспечения одинакового режима дросселирования, который соответствовал бы $\mathrm{M}_{\text {вых }}=0.348$, на выходной границе из воздухозаборника фиксировалось статическое давление $P_{\text {вых }}=86036$ Па. Все размеры отнесены к диаметру выхода В3 $d=50 \mathrm{MM}$.

\section{3. Сравнение результатов расчетов с данными эксперимента}

Анализ полученных результатов расчета с помощью ESI RANS, приведенных в работе [5], показал качественное совпадение по интегральным характеристикам с данными эксперимента. Однако картина течения в выходном сечении В3 заметно отличалось от той, которая наблюдалась в эксперименте. Были выполнены расчеты с помощью RANS/ILES метода, который явным образом описывает турбулентные вихри, что позволяет существенно повысить описания сложных турбулентных течений. При анализе результатов расчетов была выполнена верификация и валидация ESI RANS и RANS/ILES метода. Для корректного расчета в обоих пакетах использовалась одинаковая модель турбулентности - Спаларт - Аллмарас.

На рис. 2 представлены поля осредненной продольной скорости в плоскости симметрии ЛА. Хорошо видна низкоскоростная зона на фюзеляже ЛА перед входом в диффузор. Низкоскоростная зона на верхней стенке диффузора В3 распространяется вниз по потоку. Следует обратить внимание, что при расчете с помощью RANS/ILES метода низкоскоростная зона имеет меньшую длину по сравнению с расчетом ESI RANS.

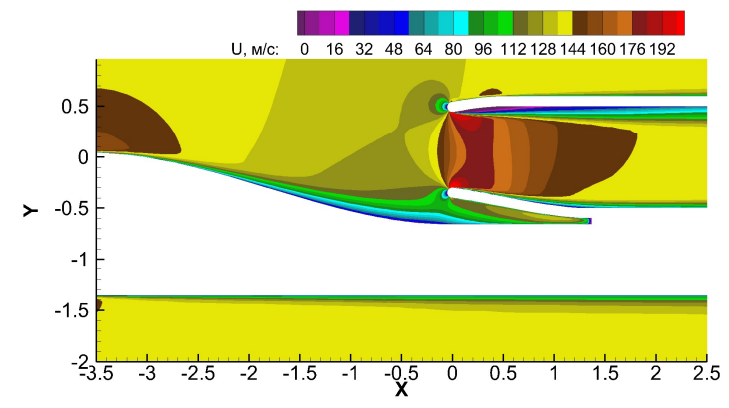

a)

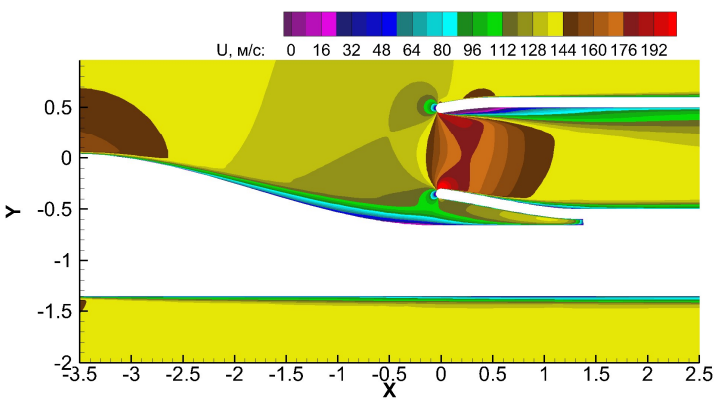

б)

Рис. 2. Поля продольной скорости в сечении плоскости симметрии:

a) ESI RANS; б) RANS/ILES метод

На рис. 3 показаны поля коэффициента восстановления полного давления в сечении выхода, полученные в эксперименте и в расчетах. Видно, что картина течения, полученная в результате расчета вихреразрешающим RANS/ILES методом, достаточно хорошо коррелирует с экспериментом. В расчетах с помощью ESI RANS наблюдаются низкоскоростные зоны в пристеночных областях диффузора, которых нет в эксперименте и в расчете с помощью RANS/ILES метода. 


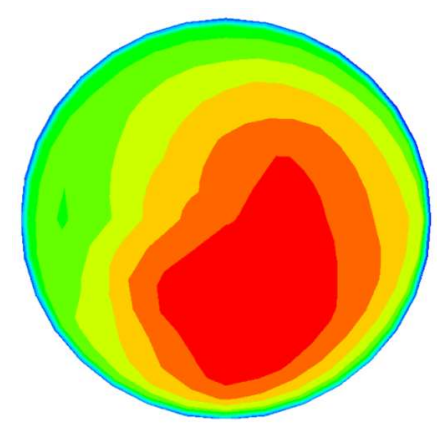

$a$

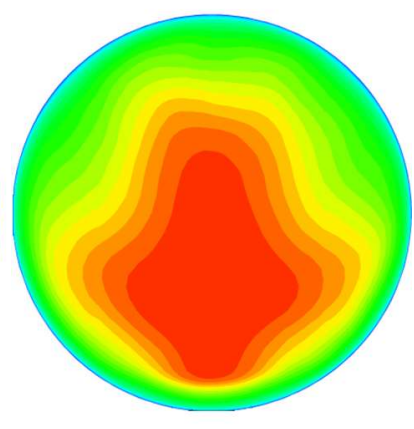

$\sigma$

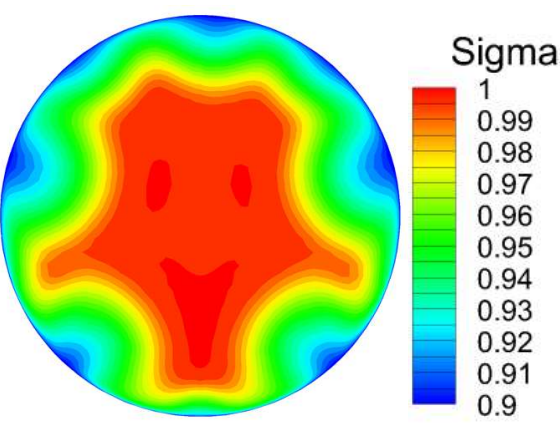

B

Рис. 3. Поля коэффициента восстановления полного давления в сечении выхода В3:

a) эксперимент; б) RANS/ILES метод; в) ESI RANS

В табл. 1 представлены интегральные характеристики на выходе из В3 для эксперимента и расчетов различными методами. Установлено, что отличие значений коэффициента восстановления полного давления, полученных этими методами, не превышает 1 \%.

\section{Таблий 1}

Параметры течения на выходе из В3

\begin{tabular}{|c|c|c|c|}
\hline & Эксперимент & RANS ESI-software & RANS/ILES \\
\hline $\mathrm{M}_{\text {вых }}$ & 0.348 & 0.3485 & 0.3465 \\
\hline$\sigma_{\text {вых }}$ & 0.972 & 0.9678 & 0.973 \\
\hline$\Delta \mathrm{M} \%$ & - & 0.14 & 0.4 \\
\hline$\Delta \sigma \%$ & - & 0.4 & 0.1 \\
\hline
\end{tabular}

Отличие структур течений, полученных с помощью RANS/ILES метода и ESI RANS, объясняется тем, что вихреразрешающие методы позволяют более точно описывать сложные турбулентные течения. С учетом этого исследование влияния синтетических струй на течение в В3 было выполнено с помощью RANS/ILES метода.

\section{4. Исследование влияния синтетических струй на течение в В3}

\section{1. Синтетические струи на поверхности фюзеляжа}

На основе анализа результатов расчетов, описанных выше, установлено, что необходимо снижение окружной неравномерности полного давления на выходе из ВЗ. На рис. 3 видно, что имеются две отрывные зоны: на поверхности фюзеляжа и на верхней стенке диффузора В3. Наличие отрывных зон может увеличивать неравномерность течения в В3. Для уменьшения неравномерности потока перед входом в В3 были использованы синтетические струи. В соответствии с рекомендациями работы [8] щели для их выхода располагались в начале отрывной зоны.

Для моделирования синтетических струй использовался упрощенный подход: на части поверхности, соответствующей щелям для выхода синтетических струй, задавалась нормальная к ней компонента скорости, которая менялась по гармоническому закону. Синтетические струи выдувались вертикально вверх. Координата начала щелей для выдува синтетических струй соответствовала $x / d=-2.1$, длина синтетических струй составляла $l / d=0.3$, ширина$h / d=0.01$. Всего рассматривалось четыре щели для выдува синтетических струй (рис. 4). На рисунке красным цветом схематично показано расположение щелей для выхода синтетических струй, а бирюзовым - сечение входа в В3. Расчетная структурированная сетка составляла $14.6 \times 10^{6}$ ячеек. Амплитуда и частота определяли режимные параметры синтетических струй. При проведении расчетов задавалась амплитуда скорости $q=100 \mathrm{~m} / \mathrm{c}$ и частота $f=100$ Гц. Граничные условия внешнего обтекания и дросселирования оставались теми же. 


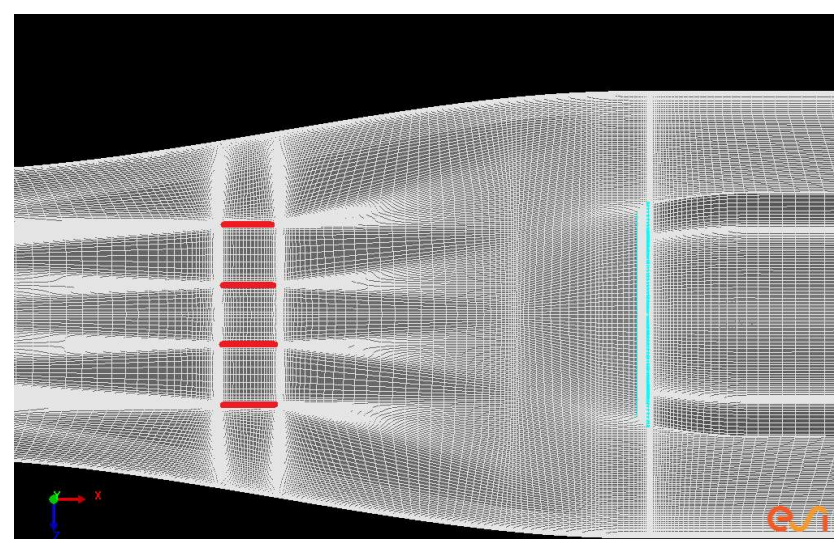

Рис. 4. Схема расположения щелей для выхода синтетических струй с поверхности фюзеляжа

Было проанализировано влияние синтетических струй на поля течений осредненной продольной скорости по длине фюзеляжа В3 рис. 5.

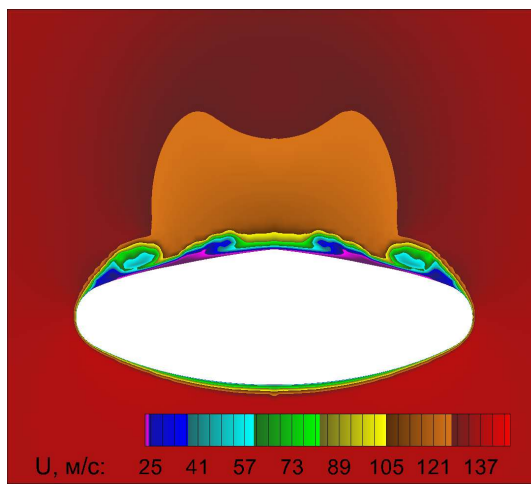

a)

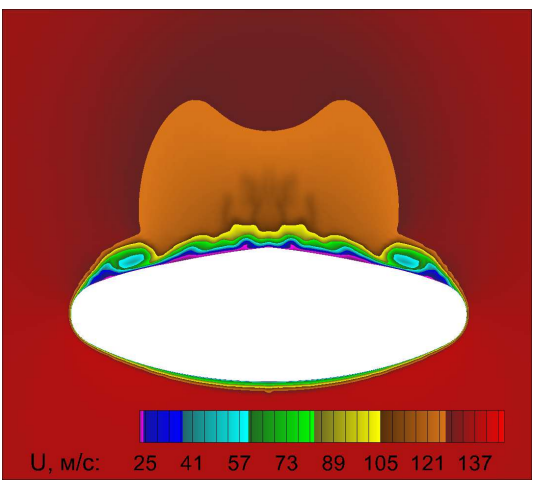

б)

Рис.5. Влияние синтетических струй на поле осредненной скорости в поперечном сечении перед входом в В3: a) без синтетических струй; b) с синтетическими струями

Видно, что синтетические струи положительно действуют на течение в области фюзеляжа ЛА, уменьшая неравномерность распределения осредненной продольной скорости в окружном направлении. Однако, изменение неравномерности на поверхности фюзеляжа перед входом в В3 не привело к улучшению параметров на выходе из него, что показано на рис. 6, где представлены поля параметров в сечении выхода диффузора.

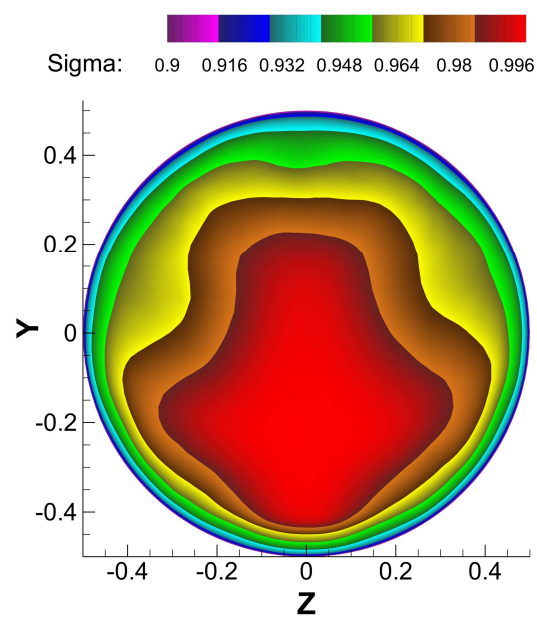

a)

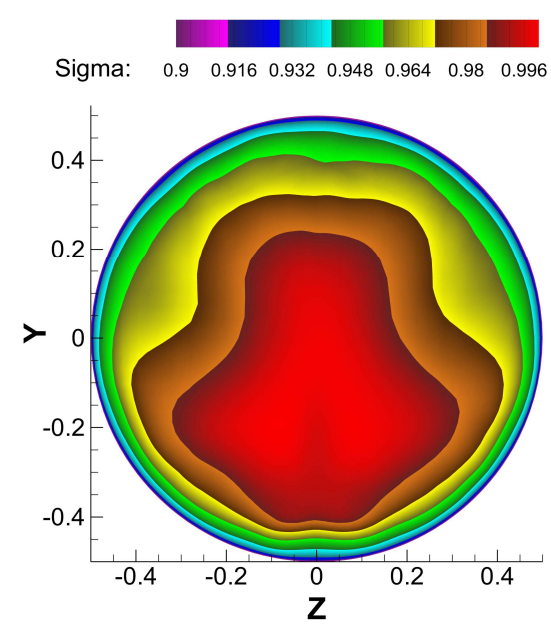

б)

Рис. 6. Влияние синтетических струй на поле коэффициента восстановления полного давления на выходе из В3: a) без синтетических струй; б) с синтетическими струями 
Это объясняется слабым взаимодействием набегающего пограничного слоя с течением внутри диффузора В3. Видно, что картина течения при использовании синтетических струй практически не меняется. Таким образом, можно сделать вывод, что основным фактором, определяющим характер течения и повышенный неравномерности течения в исследуемом В3, является особенности геометрии канала B3: переход D-образного сечения на входе в B3 в круглое на выходе из него.

\section{2. Синтетические струи внутри диффузора В3}

На основании рекомендаций [8] щели синтетических струй были за обечайкой около входа в диффузор В3 в области отрывных течений (рис 3б). Синтетические струи выдувались перпендикулярно поверхности В3 (рис. 7). Расчетная сетка содержала $18 \times 10^{6}$ ячеек.

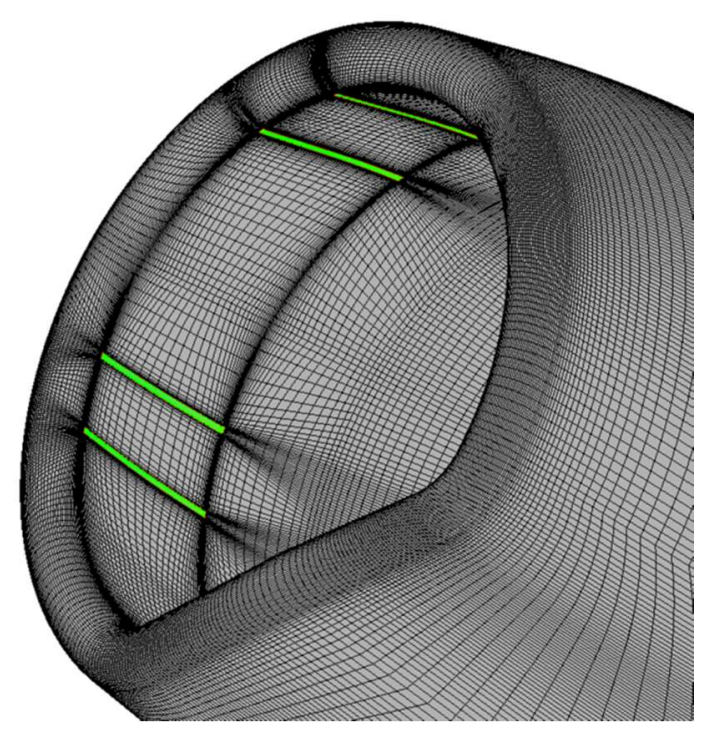

Рис. 7. Расчетная сетка и схема расположения щелей для выхода синтетических струй с поверхности В3

Амплитуда и частота определяли режимные параметры синтетических струй. В данной задаче задавалась амплитуда скорости $q$ и частота $f$ согласно табл. 2.

\section{Таблица 2}

Таблица режимов синтетических струй

\begin{tabular}{|c|c|c|}
\hline № режима & $q, \mathrm{~m} / \mathrm{c}$ & $f$, Гц \\
\hline 1 & 0 & 0 \\
\hline 2 & 50 & 100 \\
\hline 3 & 50 & 150 \\
\hline 4 & 100 & 100 \\
\hline 5 & 150 & 100 \\
\hline
\end{tabular}

Были получены поля коэффицита восстановления полного давления на выходе из В3 для различных режимных параметров синтетических струй (рис. 8). На рисунке видно, что варианты синтетических струй №№ 4 и 5 позволяют уменьшить область с пониженным уровнем коэффициента восстановления полного давления в верхней части сечения. Количественная оценка влияния синтетических струй проведена для окружной неравномерности $\Delta \sigma_{0}$. На упомянутых режимах этот параметр улучшился на $36.9 \%$ и $36.7 \%$ соответственно. Режим 2 также показал некоторое улучшение на 9.7\%, а синтетические струи № 3 вызвали ухудшение окружной неравномерности на $8.1 \%$. 

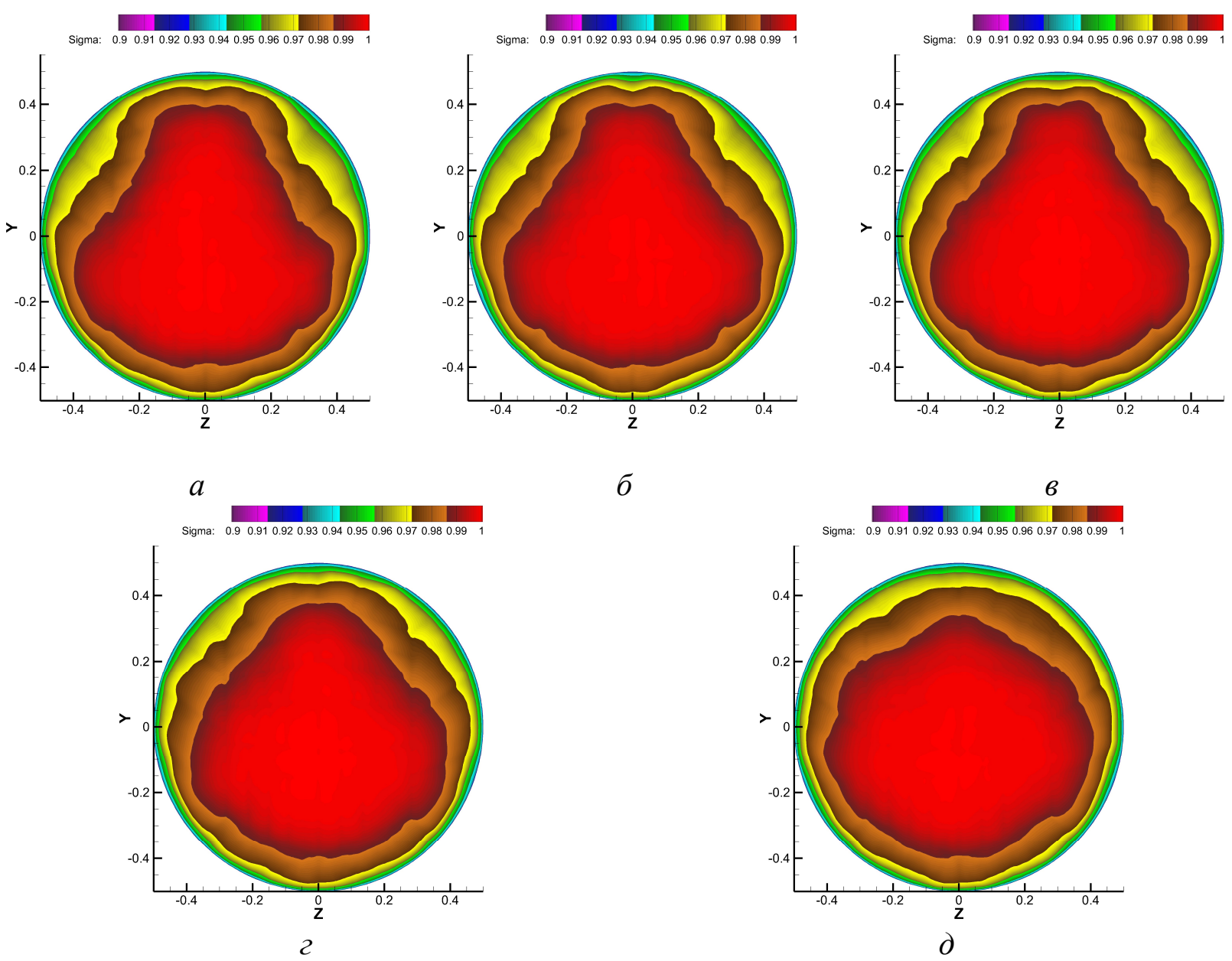

$\sigma$

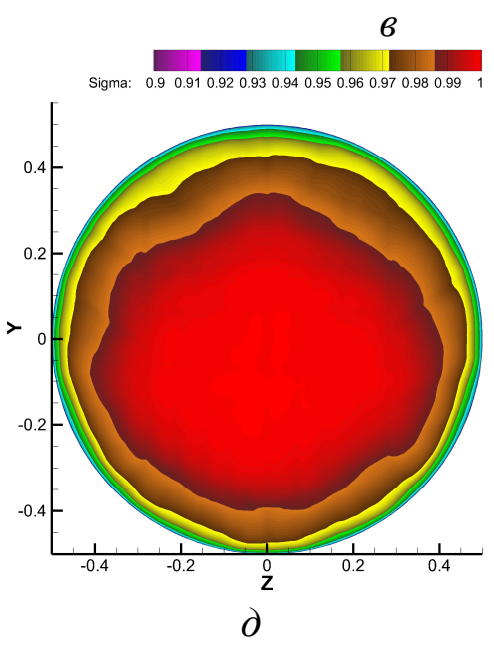

Рис. 8. Влияние режимных параметров синтетических струй на поле коэффициента восстановления полного давления на выходе из В3: a) режим $1, \sigma)$ режим 2 , в) режим 3 , г) режим $4, \partial$ ) режим 5

Количественная информация о влиянии всех исследованных вариантов синтетических струй на характеристики течения на выходе из В3 приведена в таблице 3:

\section{Таблиия 3}

Таблица значений окружной неравномерности в результате расчетов

\begin{tabular}{|c|c|c|}
\hline № режима & $\Delta \sigma_{0 i}$ & $\left(\Delta \sigma_{0 i}-\Delta \sigma_{01}\right) / \Delta \sigma_{01} \%$ \\
\hline 1 & 0.473 & 0 \\
\hline 2 & 0.427 & -9.7 \\
\hline 3 & 0.511 & 8.1 \\
\hline 4 & 0.298 & -36.9 \\
\hline 5 & 0.299 & -36.7 \\
\hline
\end{tabular}

Стоит отметить, что режимы струй, у которых амплитуда близка к скорости потока внутри канала оказавают наибольший эффект на уровень окружной неравномерности.

\section{5. Заключение}

1. Проведено исследование особенностей течения в пространственном дозвуковом В3, интегрированным с планером ЛА сложной формы при числе Маха набегающего потока $\mathrm{M}_{0}=0.4$ на нулевом угле атаки и скольжения. На выбранном режиме дросселирования $\left(\mathrm{M}_{\text {вых }}=0.348\right)$ проведено сравнение результатов расчетов с помощью ESI RANS, RANS/ILES метода с экспериментальными данными. 
2. Анализ результатов показал, что:

- К Картина течения, полученная в результате расчета вихреразрешающим RANS/ILES методом, достаточно хорошо совпадает с данными эксперимента. В расчетах с помощью ESI RANS наблюдаются низкоскоростные зоны в пристеночных областях диффузора, которых нет в эксперименте и в расчете с помощью RANS/ILES метода. Однако, различие значений коэффициента восстановления полного давления в сечении выхода В3, составляет менее $1 \%$.

- Синтетические струи на поверхности планера ЛА в области неравномерного течения позволили несколько уменьшить неравномерность распределения осредненной продольной скорости в окружном направлении перед входом в В3, однако это не повлияло на неравномерность потока на выходе из ВЗ. Это может всидетельствовать о том, что образовавшийся на планере пограничный слой перед ходом в В3 слабо влияет на параметры в выходном сечении В3.

- Синтетические струи, расположенныен в области отрывных течений внутри В3 позволили снизить уровень окружной неравномерности полного давления на выходе из В3 на $37 \%$. Стоит отметить, что данные результаты получились при амплитудных скоростях близких к потоку внутри канала.

\section{Литература}

1. Frances Fiorina, "Safety of course", Aviation week and space technology, July 28, 2008, page 70.

2. Berrier B.L., Carter M.B., and Allan B.G. High Reynolds Number Investigation of a Flush-Mounted, SDuct Inlet with Large Amounts of Boundary Layer Ingestion // NASA/TP. 2005. № 213766. 170 p.

3. Gissen A.N., Vukasinovic B., McMillan M.L., Glezer A. Distortion Management in a BLI Inlet Diffuser using Synthetic-Jet Hybrid Flow Control // AIAA P. 2011. № 35.

4. Gissen A.N., Vukasinovic B., Glezer A. Manipulation of Stream wise Vorticity in an Emulated Diffuser Boundary Layer Using Hybrid Flow Control // AIAA P. 2010. № 4586.

5. Постников А.А., Виноградов В.А., Комратов Д.В., Степанов В.А., Скрябин А.С. Исследование процессов обтекания дозвукового входного устройства, интегрированного с планером летательного аппарата// Физико-химическая кинетика в газовой динамике. 2018. T.19, вып. 4. http://chemphys.edu.ru/issues/2018-19-4/articles/756/

6. ESI GROUP CFD - ACE.

7. Любимов Д.А. Разработка и применение метода высокого разрешения для расчета струйных течений метод крупных вихрей // ТВТ. 2012. Т. 50. №3. С. 450-466.

8. Любимов Д.А., Потехина И.В. Применение RANS/ILES-метода для анализа эффективности управления отрывными течениями в диффузорах с помощью синтетических струй // МЖГ. 2015. № 4. C. 144-154. 